\title{
The scaling of relative task difficulty across spatial, brightness, and form successive discrimination reversal (SDR) problems with Capuchin monkeys
}

\author{
ROBERT L. GOSSETTE AND HARVEY R. BROWN \\ COMPARATIVE ANIMAL BEHAVIORAL LABORATORIES HOFSTRA UNIVERSITY
}

The successive reversal performances of Capuchin monkeys assigned to spatial, brightness, and form discrimination tasks were compared to determine relative level of difficulty of each task. Comparison of error, session, and initial error measures all indicated that spatial and form tasks were respectively the easiest and most difficult, with brightness intermediate.

While many standard learning tasks such as classical conditioning, avoidance, and simple discrimination (Warren, 1965) have typically proved unsuccessful in yielding interspecies differences in any systematic, taxonomically meaningful fashion, two closely related procedures-learning set (LS) and successive discrimination reversal (SDR)-have shown considerable promise. LS, though useful among higher mammals, appears to have little usefulness among the lower mammals and submammalian species. SDR, however, appears applicable across wide ranges of vertebrates (Bitterman, 1965). Among birds, SDR analysis not only reveals appreciable interspecies differences, but indicates that the magnitude of such differences is importantly related to the degree of interspecies remoteness, with closely related species displaying more similar error functions than distantly related species (Gossette, Gossette, \& Riddell, 1966; Gossette, 1966). Among mammals, spatial SDR analysis has also shown promising resolution of performance differences among various members of the raccoon family and between squirrel and Capuchin monkeys (Gossette \& Inman, 1966; Gossette \& Speiss, 1967). However, the superior performance of the Capuchin monkeys suggests that differentiation among the higher primates might be difficult due to the relative ease with which minimal error reduction on spatial SDR is achieved. Previous study with birds indicated that task difficulty, e.g., errors across reversal problems, increased greatly as training proceeded from a spatial to a brightness SDR (Gossette, 1967). Still greater errors were produced by these $\mathrm{Ss}$ on a subsequent form SDR. Importantly, the small interspecies differences of closely related species obtained on the spatial SDR were progressively amplified by the brightness and form SDRs. Thus it appears that one way of controlling the "resolving power" of SDR calibration is through control of task difficulty. This should facilitate interspecies comparisons among higher primates.

Accordingly, as an initial step in scaling relative
SDR task difficulty, the reversal performances of Capuchin monkeys on a spatial, a brightness, and a form task were analyzed.

Method

Ten Capuchin monkeys were assigned as follows: five to a spatial, three to a brightness, and two to a form task. All Ss had been maintained in the vivarium for at least three months prior to testing. Three weeks prior to testing each $S$ was placed on a $23 \mathrm{~h}$ deprivation schedule, and habituated to the test chamber. The apparatus consisted of a formboard box previously described (Gossette \& Inman, 1966). Two round white blocks, a pair of black and white round blocks, and a pair of square and round black blocks served as stimuli, respectively, for the spatial, brightness, and form reversal tasks. Each $S$ was trained to dislodge the appropriate set of blocks to retrieve a small piece of banana from the foodwells. Equal training was provided with each block-position combination. The initial habit, Problem 1, was established. When a criterion of two or fewer errors on a given day was achieved, reversal training was initiated and continued until criterion was again achieved. A total of 19 reversals were obtained. Positioning of the stimulus blocks for the brightness and form experiments was randomly varied within each daily session of 20 trials. Non-correction was employed.

Results

Three major measures of reversal performance were selected for analysis: total errors, sessions, and initial errors, across the 20 problems (see Table 1). When analyzed by Kruskal-Wallis test, differences among the three task conditions were significant for all measures $(p<.01)$. The tasks studied clearly sample different levels of task difficulty, with the form task producing an appreciable elevation of error scores. Figure 1 shows the error reduction functions for each group across the 20 problems. The curves for the

Table 1

Mean total errors, sessions and initial errors across 20 problems.

\begin{tabular}{lccc}
\hline Task & $\begin{array}{c}\text { Measures } \\
\text { Errors }\end{array}$ & Sessions & Initial Errors \\
\hline Form & 531 & 88 & 82 \\
Brightness & 202 & 45 & 53 \\
Spatial & 51 & 26 & 23 \\
\hline
\end{tabular}




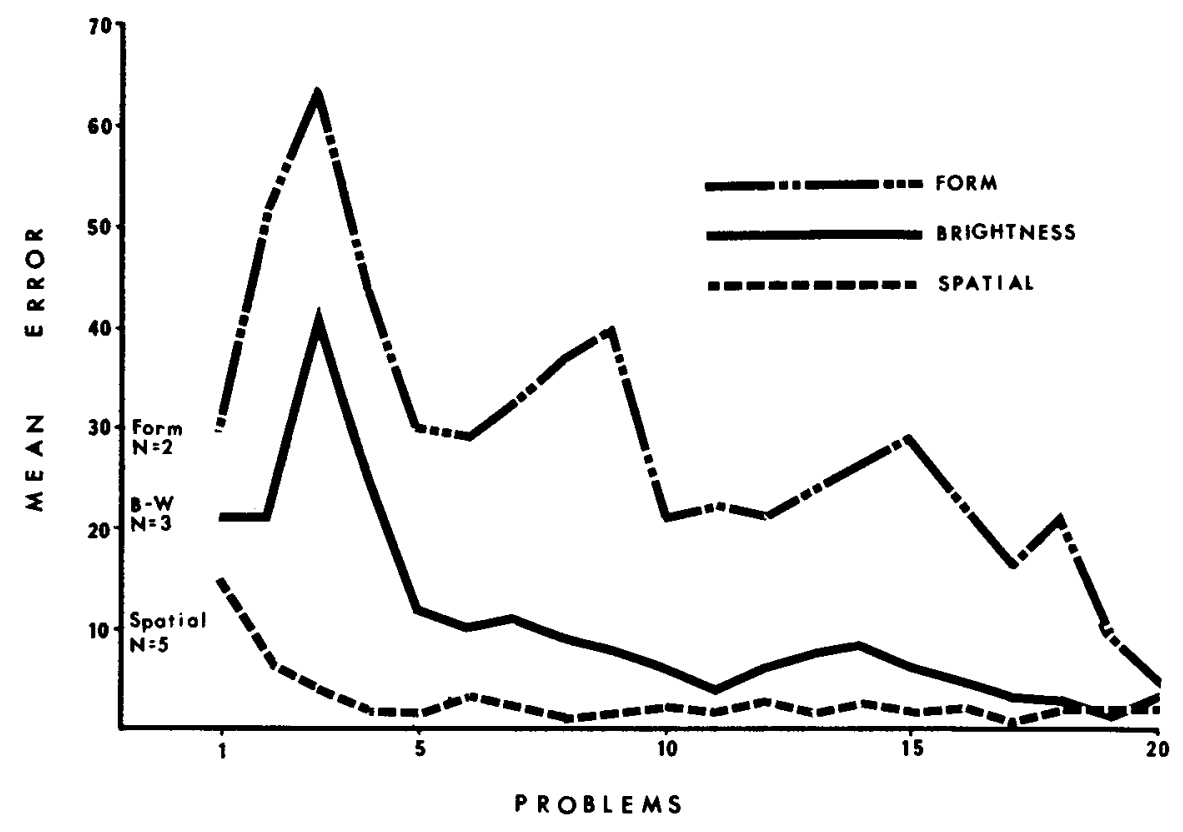

Fig. 1. Mean error per problem on spatial brightness, and form successive reversal tasks. other two measures are essentially similar. Of particular interest is the appearance of peak error score on Problem 1, the original habit, for the spatial group, while the form and brightness groups show a peak error on the second reversal, Problem 3. Much of the SDR literature, especially that employing rats, has reported peak error on the first reversal. Recently, Gossette \& Gossette (1967) have suggested that the location of peak error may well be a significant dependent variable of taxonomical import, since "lower order" birds tend to produce a peak error that is displaced to later reversals than that of "higher order"' birds. The present data suggest that location of peak error may also be controlled by task difficulty. Consideration of the variables controlling this measure becomes critical in any attempt to utilize the reversal index (RI) proposed by Rajalakshim \& Jeeves (1965) in attempting to build a behavior taxonomy. Finally, it should be noted that task difficulty, while determining the rate of error reduction, did not generate any appreciable difference in asymptotic error scores. All three groups achieved a one error per problem solution score by Problem 20 .
References

BITTERMAN, M. E. The evolution of intelligence. Scient. American, 1965, 212, 92-100.

GOSSETTE, R. L. Comparison of successive discrimination reversal performances across 14 different avian and mammalian species. Amer. Zool, 1966, 6, 545 (abstract).

GOSSETTE, R. L. Successive discrimination reversal (SDR) performances of four avian species on a brightness discrimination task. Psychon. Sci, 1967, 8, 17-18.

GOSSETTE, R. L., \& INMAN, N. Comparison of spatial successive discrimination reversal performances of two groups of New World monkeys. Percept. mot. Skills, 1966, 23, 169-170.

GOSSETTE, R. L., GOSSETTE, M., \& RIDDELL, W. Comparisons of successive discrimination reversal performances among closely and remotely related avian species. Anim. Behav., 1966, 14, 560-564.

GOSSETTE, R. L., \& SPEISS, J. Comparison of SDR performances of seven mammalian species on a spatial task. Submitted for publication, 1967.

RAJALAKSHMI, R., \& JEEVES, M. The relative difficulty of reversal learning (reversal index) as a basis of behavioral comparisons. Anim. Behav., 1965, 13, 203-211.

WARREN, J. M. Comparative psychology of learning. In Annu. Rev. Psychol. Vol. 16. Palo Alto, California: Annual Reviews, Inc., 1965.

\section{Note}

1. Supported in part by NIMH grant No. MH 11806-01 awarded to the senior experimenter. 